\title{
An Improved K-view Algorithm for Texture Classification Using Voting Method
}

\author{
Changwang Liu ${ }^{1}$, Jianfang Wang ${ }^{*}, 2$, Zhidu Liu ${ }^{2}$, Anfeng $\mathrm{Xu}^{2}$ and Yihua Lan ${ }^{2,3}$ \\ ${ }^{1}$ School of Software, Nanyang Normal University, Nanyang, 473061, China \\ ${ }^{2}$ School of Computer and Information Technology, Nanyang Normal University, Nanyang, 473061, China \\ ${ }^{3}$ School of Computer Science and Technology, Huazhong University of Science and Technology, Wuhan, 430074, China
}

\begin{abstract}
Texture is an important characteristic of images. Image texture classification is widely used in many applications, such as detection of industry product, document class, and digital image processing of remote sensing and so on. Several K-View based algorithms have been proposed for image texture classification. This study proposed an improved k-view algorithm for texture classification using voting method. In the first step, the method was implemented in our previous study that all the views used are transformed into rotation-invariant features is employed. Then $\mathrm{K}$ views are selected randomly as those characteristic views to form characteristic view sets for each class texture. In the third step, we use those characteristic view sets to class a texture image and achieve a preliminary result. At last, voting method is used to improve the preliminary result. A lot of experiments are carried out using artificial texture images taken from the Brodatz. The experimental results showed that he proposed improved method is more robust and accurate by compared with the old method.
\end{abstract}

Keywords: Texture classification, characteristic views, k-view-template algorithm, k-view-datagram algorithm, voting algorithm.

\section{INTRODUCTION}

Texture is an important characteristic of objects surface and can be seen everywhere in our daily living, some familiar texture images are shown in Fig. (1). The aims to class a texture image is that expecting the computer can class the different texture classes auto just like as human, make the subsequent work such as object recognition, analysis and tracking are possible. Since the image regions usually take on texture characteristics, the different image regions may be recognized by using texture, therefore, the technique related to texture image classification are used wcidely, take the remote sensing image analysis for example, it always used spectrum information to class different objects on ground such as grass, ocean and building and so on, however, there are phenomenon such as "different objects have same spectrum" or "same objects have different spectrum", the classification accuracy is not satisfactory in the case of use spectrum feature singly. But the different objects on ground usually have different texture features, by using spectrum feature and texture features at the same time, we may be improve the classification accuracy [1]. Along with the diagnosis or assistant diagnosis is more and more depend on the medical image technique such as ultrasonic, $\mathrm{X}$ radial, CT, MR and so on, the texture features are looked as important characteristics and used in the medical image processing [24].The texture image classification technique is also used in the quality detection of industry product, document class and image retrieval based on content etc.

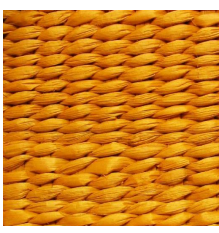

(a) pillow texture

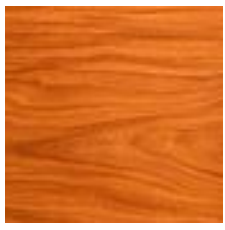

(c) board texture

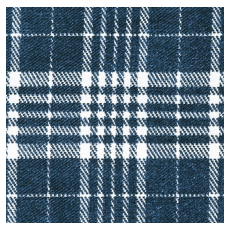

(e) cloth texture

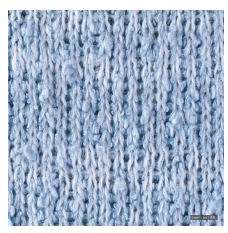

(b) sweater texture

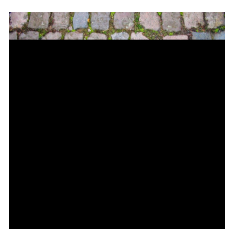

(d) dust wall texture

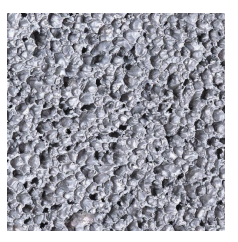

(f) black metal texture
Fig. (1). Familiar texture images.

Although it is sure that people may perceive the presence of texture, but there is still no authoritative definition of the 
texture itself, at the same time, the classification method always different in different application, therefore, until now, the texture classification is still a hotspot and a difficulty of in the field of machine vision and image analysis, there are many questions need to be further researched and solved.

\section{RELATED WORKS}

According to the number of texture classes whether or not known, the texture classification technique may be classed to supervised methods and unsupervised methods [56]. In despite of that the unsupervised method is more useable than supervised method, but the determination of the number of texture classes is a difficulty, the unsupervised method is not easy to implement, therefore, the researches on supervised method are more. Despite the supervised method or unsupervised method, image texture classification methods can be divided into two major categories; the first category is based on features with a high degree of spatial localization, such as with some form of edge detection operation. The major problem with this type of approach is that it is difficult to distinguish the texture boundaries and the microedge located in the same texture. The second category is based on discrimination functions using several texture features. The classification accuracy in this approach depends upon the discriminative power of the texture features. The most important step for the second approach is to extract texture features, which can discriminate different types of texture in the original image. Many methods have been developed to describe texture features. there are usually model analysis methods [7-10], spectrum analysis methods [11-13], statistics methods [14-16] and structure methods [17-18] etc.

Hung et al proposed K-View-Template algorithm and KView-Datagram algorithm based on characteristic views [19], these algorithms have achieved reasonable classification accuracy by contrast with other methods, but there are inherent shortcomings, such as to extract characteristic Views by using K-Means method or FCM method which may be need many iterative computation, the expense on processing time is very large, especially the K-ViewDatagram algorithm, needs to calculate corresponding Datagram of each sub-image which will be classified, the calculation work may be huge, in addition, the characteristic views are rather the average of the primary view set than original information of each texture classes and can not be represent the each original texture well. In this way, Hong Liu et al proposed K-View using rotation-invariant feature algorithm [20] and Fast Weighted K-View-Voting Algorithm [21] to improve the original two K-View algorithms by using different approaches and achieved more good classification accuracy. In this paper, we proposed an improved $\mathrm{K}$ view algorithm based on the K-View using rotation-invariant feature algorithm. By using voting method, the improved method is more robust and accurate.

The rest of this paper is organized as follows: in section 3, the K-View using rotation-invariant feature algorithm is briefly introduced; the improved algorithm using voting method is detailed in section 4 . Then give the experiments and results in section 5, and analysis are also given in this section 5; the conclusions are given in section 6 then.

\section{THE K-VIEW USING ROTATION-INVARIANT FEATURE ALGORITHM}

The K-View Using Rotation-Invariant Feature algorithm consists of two processes which are the training process and the classification process. The scheme of each process is shown in Fig. (2) and Fig. (3) respectively [21].

There are two enhancements to improve the original $\mathrm{K}$ View-Template algorithm and the K-View-Datagram algorithm in K-View Using Rotation-Invariant Feature algorithm.

\subsection{The Method for Extracting "Characteristic Views Set"}

In the original $\mathrm{K}-\mathrm{View}$-Template algorithm and the $\mathrm{K}$ View-Datagram algorithm, the characteristic views can be learned by using the unsupervised clustering algorithms such as the k-means or fuzzy c-means methods. Different from the above two K-View algorithms, the characteristic views are randomly and directly selected from the view set of "Kernel" in each texture class in the K-View Using Rotation-Invariant Feature algorithm. By compare with the old selecting method in origin K-view algorithms, the benefit of this method is that it can keep the primitive information of views as many as possible.

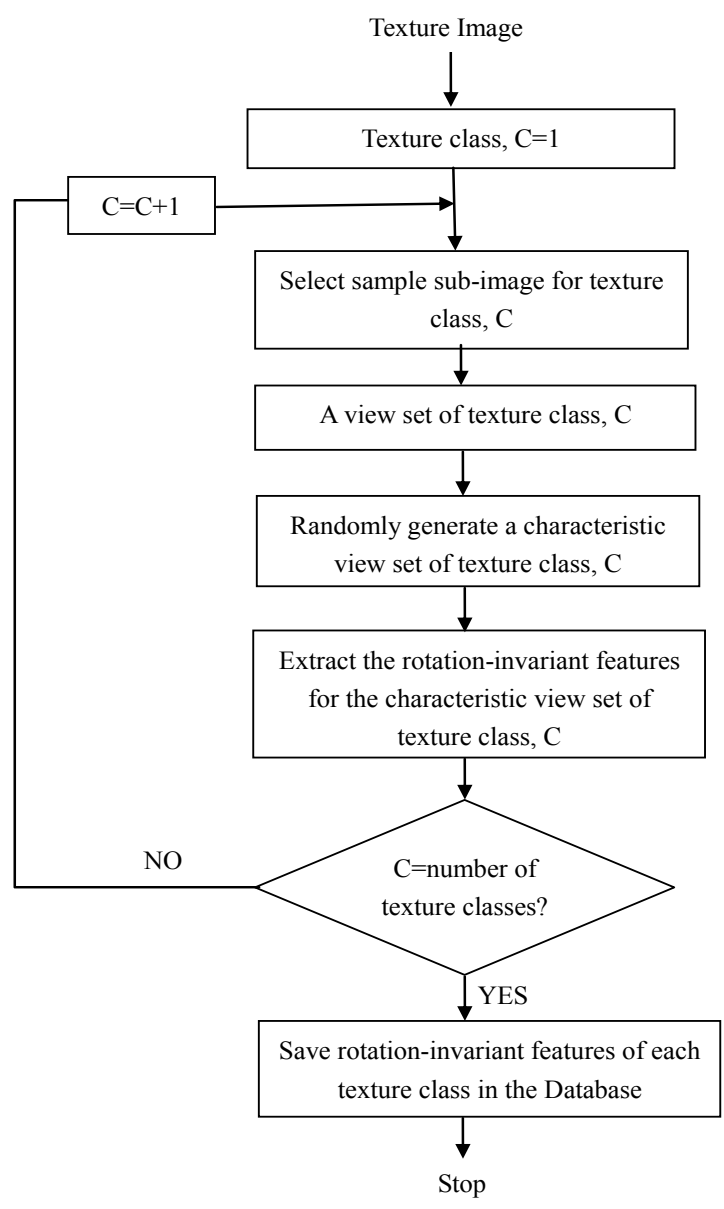

Fig. (2). The training process of this scheme. 


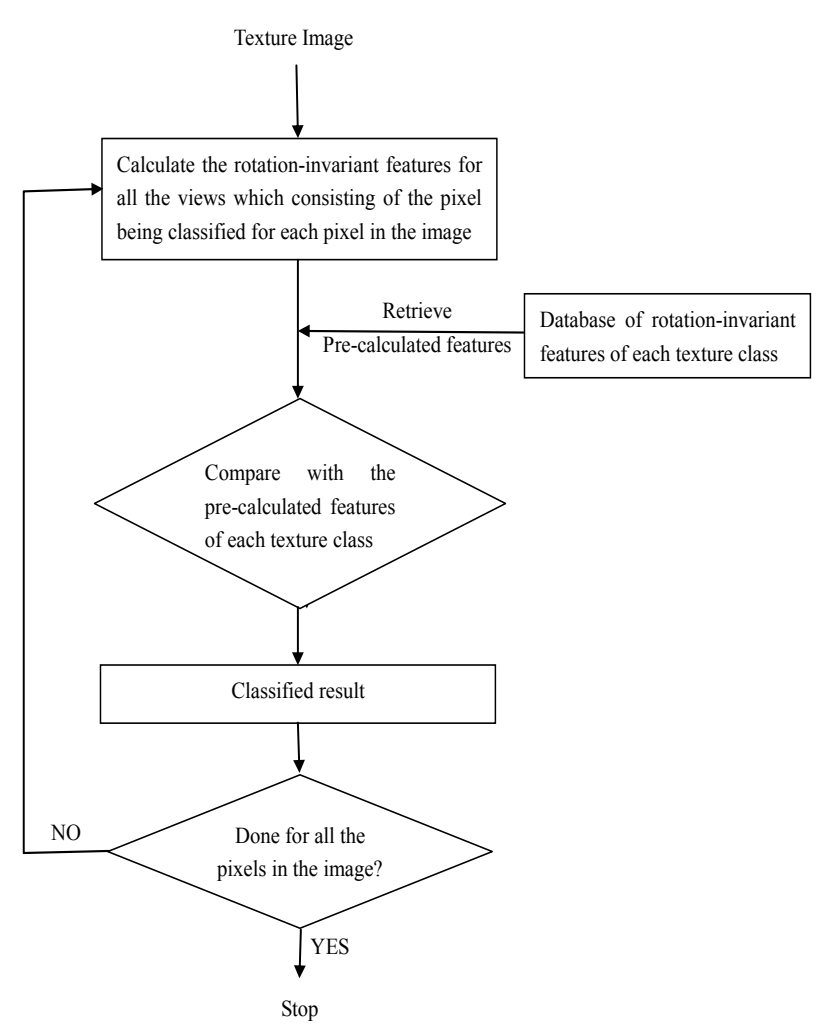

Fig. (3). The classification process of this scheme.

\subsection{The Correlation-Matching Method Between Views}

The K-View-template algorithm compare the similarity between a view and all "characteristic views" directly, while the K-View-datagram algorithm compute the histogram distribution of the "characteristic views" for each texture class and the comparison is transformed into between the histogram of the view and the histograms of each texture class. In the K-View Using Rotation-Invariant Feature algorithm, in order to achieve higher classification accuracy, six significant rotation-invariant features named Mean, Standard Deviation, Entropy, Skew, Kurtosis and Histogram are extracted. All the characteristic views used are transformed into rotation-invariant features, which provide a good discrimination of textures. The rotation-invariant features of a view whose pixel number is $\mathrm{N}$ in the following:

(1) Feature 1: Mean

$\bar{x}=\frac{x_{1}+x_{2}+\wedge+x_{n}}{n}=\frac{1}{n} \sum_{i=1}^{n} x_{i}$

Where, $V_{i}$ is the gray value of the ith pixel in the view.

(2) Feature 2: Standard Deviation

$S=\left[\frac{1}{N-1}\left(\sum_{i=1}^{n} x_{i}^{2}-n \bar{x}^{2}\right)\right]^{\frac{1}{2}}$

Where, $\bar{V}$ is the mean of the view.

(3) Feature 3: Entropy
$x_{3}=-\sum_{i=1}^{N} p_{i} * \ln p_{i}$

(4) Feature 4: Skew

$S=\frac{M_{3,3}}{\delta^{3}}$

Where, $M_{3,3}$ is the third-order moments of the view, $\delta$ is the standard deviation of the view.

(5) Feature 5: Kurtosis

$K=\frac{M_{4,4}}{\delta^{4}}$

Where, $M_{4,4}$ and $\delta$ is respectively the fourth-order moments and the standard deviation of the view.

The sixth feature is histogram. Then the six rotationinvariant features are formed into a normalization rotationinvariant feature vector, and may be used to represent different types of textures.

The correlation-matching method between views can be described as follows.

Euclidean distance was employed to measure the similarity between two views in this study. The distance between a view $(\mathrm{Xi})$ and another one view $(\mathrm{Yj})$ can be computed as following equation 6 :

$d\left(X_{i}, Y_{j}\right)=\sqrt{\sum_{r=1}^{u}\left(f_{r}\left(X_{i}\right)-f_{r}\left(Y_{j}\right)\right)^{2}}, f_{r} \in F(r=1,2, \cdots 6)$

Where $d\left(X_{i}, Y_{j}\right)$ is represented the distance between the two views. $f_{r}$ is the value of a feature form the feature vector which contains 6 features.

And then the similarity $\operatorname{Sim}\left(X_{i}, Y_{j}\right)$ between view (Xi) and view $(\mathrm{Yj})$ can be defined as follows:

$\operatorname{Sim}\left(X_{i}, Y_{j}\right)=\frac{1}{d\left(X_{i}, Y_{j}\right)}(7)$

From the equation 7 we can find that if the distance $d\left(X_{i}, Y_{j}\right)$ between two views is smaller, it means that they are more similar.

\section{THE IMPROVED ALGORITHM USING VOT- ING METHOD}

\subsection{The Concept of Promising Neighborhood Supporters}

We can use various strategies to make a decision on texture image classification. In the previous K-View-T algorithm, the decision is made by one view whose center located at the current pixel being classified while in the K-View$\mathrm{D}$ algorithm the decision is made by the distribution of all 
the views. The preliminary experimental results show that a group decision is more robust than an individual one. For example, to give a verdict of whether the dinosaurs are human's ancestors by a jury composed of archaeologists, chemists and musicians. Since archaeologists have the best knowledge in this domain, they should have the highest voice (i.e. a higher weighting factor), the chemists may have very limited knowledge about this domain and should have much less voice (i.e. a lower weighting factor), and musicians, who know very little about this domain should have the lowest voice or disclaim the voting rights (i.e. a near-zero weighting factor). With this assumption, the correct verdict would be more trustworthy. In our improved algorithm, we consider use another reasonable group called promising neighborhood supporters as the decision makers. In that method, the fittest texture class of the pixel being considered is determined by all the views in the promising neighborhood.

\subsection{Group Decision Made by Voting}

From both a theoretical and a practical research, the voting method plays an important role in the group decision [22-25]. The most natural voting strategy is simple majority. In this strategy, each voter takes a full vote on a candidate it supports, and then the candidate with the most votes is the winner. In the application of image texture classification, one is to determine which texture class a pixel belongs to. We may consider that the texture class having the majority views in the promising neighborhood supporters may be considered as the fittest one to the pixel. However, the simple majority voting strategy neglects the weighting factor of each supporter, which is sometimes not reasonable. Take Fig. (4) for example, views V1, V2, V3 and V are four promising neighborhood supporters of the red pixel. For this pixel, V shoud be have large vote than other three ones in decision the pixel is classed into texture $\mathrm{T} 2$, because $\mathrm{V}$ is more similar to $\mathrm{T} 2$ than $\mathrm{V} 1 \sim \mathrm{V} 3$.

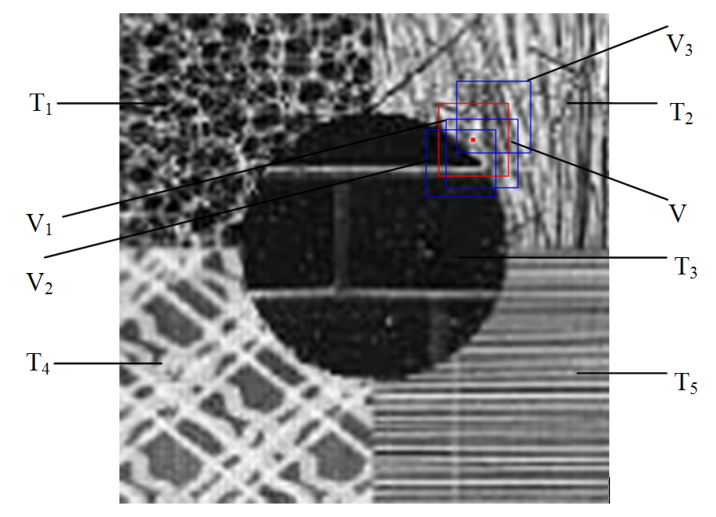

Fig. (4). An example for voting.

In our algorithm, let $d_{i j}$ be the distance, the computing method of it is same as equation (6). Then the similarity $\operatorname{Sim}\left(X_{i}, Y_{j}\right) s$ between a view and a texture class can be defined as the formula (8):

$$
\operatorname{Sim}\left(X_{i}, Y_{j}\right)=\frac{1}{d^{2}\left(X_{i}, Y_{j}\right)}
$$

From Fig. (4), we can conclude that if a view is more similar to a certain texture class, it should be take more powerful vote on the classification. For example, view $\mathrm{V}_{3}$ is more similar to $\mathrm{T}_{2}$ than V2. Hence, we define the weighting factor for $i t h$ view to $j$ th class as follows:

$$
w_{i j}=\frac{\operatorname{Sim}\left(X_{i}, Y_{j}\right)}{\sum_{j=1}^{N} \operatorname{Sim}_{j}\left(X_{i}, Y_{j}\right)}
$$

Where $N$ is the number of texture classes. View $Y_{j}$ is a characteristic view which is one of the most similar to $X_{i}$. In the other word, the similarity between view $X_{i}$ and view $Y_{j}$ represents the similarity between view $X_{i}$ and $j$ th texture class. Let each view in the pixel' (i.e., being classified) promising neighborhood supporters take the weighting factor $w_{i j}$ as a vote to make a group decision. By this way, we can compute $j$ vote sums for $j$ texture classes. The pixel being classified belongs to the class which achieves the largest sum.

\section{EXPERIMENTAL RESULTS AND DISCUS- SION}

In order to test and compare the performances of the orange and the improved K-View algorithms, a lot of experiments are carried out. To make the experimental results are more representative, we run $\mathrm{K}-\mathrm{View}$ algorithms on the images obtained randomly from Brodatz Gallery, from the original images, we may find that the artificial images consist of many kind of basal texture images which are selected randomly from Brodatz Gallery. A variety of representative texture images includes coarse texture. Some examples are listed as follows.
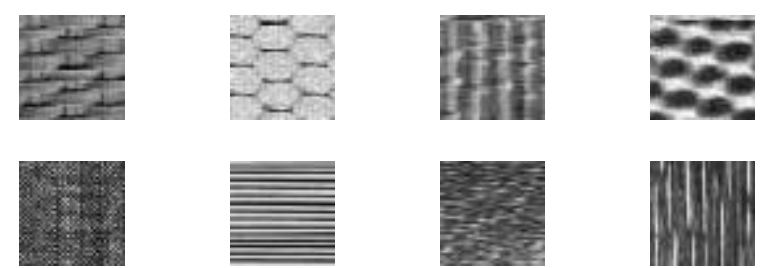

Fig. (5). Some texture images used in this study.

Fig. (6) shows some classified results of a texture images with original and improved K-View algorithms. Classified right ratios of each texture image classification with different k-view algorithms are detailed in Fig. (7). 


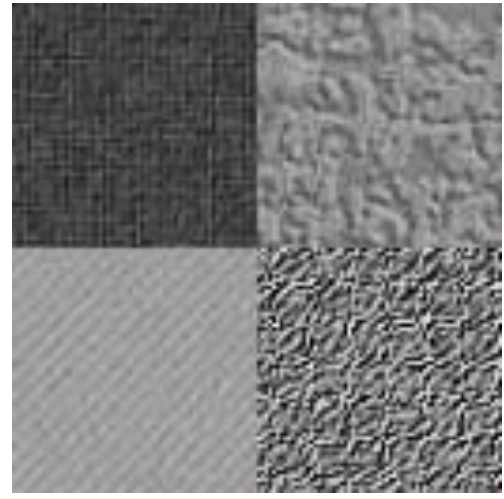

(a)

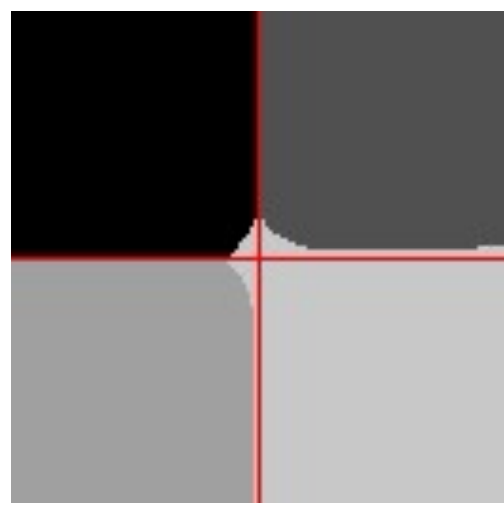

(b)

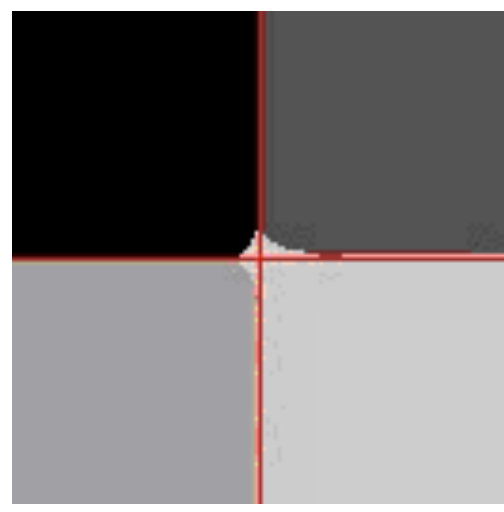

(c)

Fig. (6). The classified results of a texture images with original and improved K-View algorithms respectively.

By comparing these experimental results as shown in Fig. (6) and Fig. (7) we can see that our improved K-View algorithm using voting method performs better than the previous K-Views algorithm using rotation-invariant feature.

(a) an original image, (b) a classified result with the original K-View algorithm (i.e., K-View using rotation-invariant feature algorithm ), (c) a classified result with the improved $\mathrm{K}-\mathrm{View}$ algorithm. The red lines are drawn on the top of classified results to show the actual boundary.

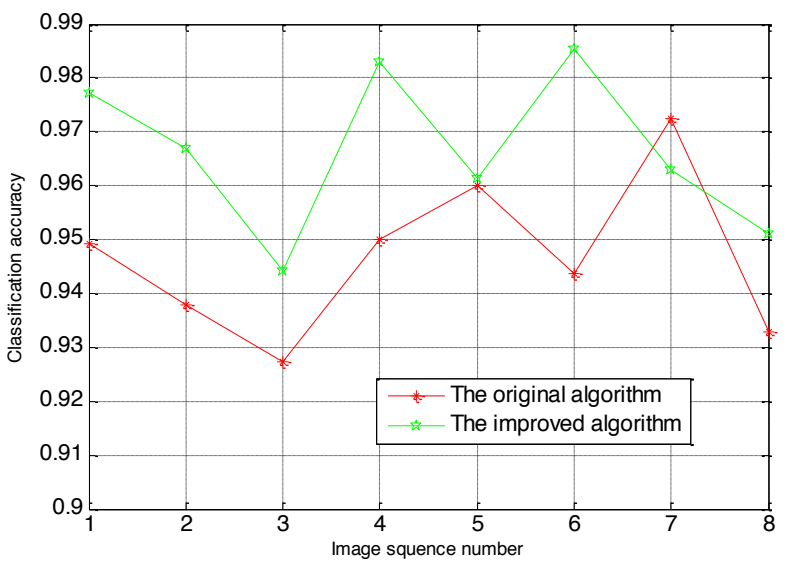

Fig. (7). Classified right ratios of each texture image classification with different k-view algorithms.

\section{CONCLUTION}

In this paper, we report an improved $\mathrm{K}$ view algorithm based on the K-View using rotation-invariant feature algorithm. Different form the old decision method which used single view to determine the classification of the pixel located in the view center in the origin algorithm, the improved algorithm employed group decision method which uses all the views in the promising neighborhood of a pixel as decision makers to determine the classification of the pixel. It is more reasonable. Experimental results show the effective of the improved algorithm.

\section{CONFLICT OF INTEREST}

The authors confirm that this article content has no conflicts of interest.

\section{ACKNOWLEDGMENT}

This study was supported in part by the National Natural Science Foundation of China (Grant No. 61401242), the Research Foundation for Advanced Talents of Nanyang Normal University (ZX2014058), Technology Research and Development Program of Lianyungang (Grant No.SH1223), the Research plan for Basic and frontier Research of Henan (Grant No. 142300410044), and the Key Programs of Education Department of Henan Province (Grant No. 14A520057).

\section{REFERENCES}

[1] C. Zhu and X. Yang, "Study of remote sensing image texture analysis and classification using wavelet," International Journal of Remote Sensing, vol. 19, pp. 3197-3203, 1998.

[2] R. N. Sutton and E. L. Hall, "Texture Measures ror Automatic Classification of Pulmonary Disease," IEEE Transactions on Computers, vol. 100, pp. 667-676,1972.

[3] D. Jirak, M. Dezortová, P. Taimr, and M. Hájek, "Texture analysis of human liver," Journal of Magnetic Resonance Imaging, vol. 15 , pp. 68-74, 2002.

[4] L. Jun-Wei, F. Huan-Qing, Z. Ying-Yue, and L. Chuan-Fu, "A novel automatic extraction method of lung texture tree from HRCT images," Acta Automatica Sinica, vol. 35, pp. 345-349, 2009. 
[5] A. Laine and J. Fan, "Texture classification by wavelet packet signatures," Pattern Analysis and Machine Intelligence, IEEE Transactions on, vol. 15, pp. 1186-1191, 1993.

[6] J. L. Chen and A. Kundu, "Unsupervised texture segmentation using multichannel decomposition and hidden Markov models," Image Processing, IEEE Transactions on, vol. 4, pp. 603-619, 1995.

[7] S. E. Grigorescu, N. Petkov, and P. Kruizinga, "Comparison of texture features based on Gabor filters," Image Processing, IEEE Transactions on, vol. 11, pp. 1160-1167, 2002.

[8] M. K. Bashar, T. Matsumoto, and N. Ohnishi, "Wavelet transform-based locally orderless images for texture segmentation," Pattern Recognition Letters, vol. 24, pp. 2633-2650, 2003.

[9] S. Arivazhagan and L. Ganesan, "Texture classification using wavelet transform," Pattern recognition letters, vol. 24, pp. 1513$1521,2003$.

[10] H. Greenspan, S. Belongie, R. Goodman, and P. Perona, "Rotation invariant texture recognition using a steerable pyramid," In: Proceedings of the $12^{\text {th }}$ IAPR International. Conference, pp. 162$167,1994$.

[11] D. C. He and L. Wang, "Texture features based on texture spectrum," Pattern Recognition, vol. 24, pp. 391-399, 1991.

[12] J. K. Beddow, Image analysis sourcebook: American Universities Science and Technology Press, 2000.

[13] H. S. Mahmood, W. B. Hoogmoed, and E. J. van Henten, "Proximal Gamma-Ray Spectroscopy to Predict Soil Properties Using Windows and Full-Spectrum Analysis Methods," Sensors, vol. 13, pp. 16263-16280, 2013

[14] M. Partio, B. Cramariuc, M. Gabbouj, and A. Visa, "Rock texture retrieval using gray level co-occurrence matrix," in Proc. of 5th Nordic Signal Processing Symposium, 2002.

[15] C. Palm, "Color texture classification by integrative cooccurrence matrices," Pattern recognition, vol. 37, pp. 965-976, 2004.
[16] C. Avilés-Cruz, R. Rangel-Kuoppa, M. Reyes-Ayala, A. Andrade-Gonzalez, and R. Escarela-Perez, "High-order statistical texture analysis - font recognition applied," Pattern Recognition Letters, vol. 26, pp. 135-145, 2005.

[17] L. Lefebvre and P. Poulin, "Analysis and synthesis of structural textures," Graphics Interface, pp. 77-86, 2000.

[18] R. M. Haralick, "Statistical and structural approaches to texture," Proceedings of the IEEE, vol. 67, pp. 786-804, 1979.

[19] S. Yang and C.-C. Hung, "Image texture classification using datagrams and characteristic views," In: Proceedings of the 2003 ACM symposium on Applied computing, pp. 22-26, 2003.

[20] H. Liu, Y. Lan, Q. Wang, R. Jin, E. Song, and C.-C. Hung, "Fast weighted $\mathrm{K}$-view-voting algorithm for image texture classification," Optical Engineering, vol. 51, pp. 027004-1-027004-12, 2012.

[21] H. Liu, S. Dai, E. Song, C. Yang, and C.-C. Hung, "A new KView algorithm for texture image classification using rotationinvariant feature," In: Proceedings of the 2009 ACM symposium on Applied Computing, pp. 914-921, 2009.

[22] S. J. Brams and M. R. Sanver, "Critical strategies under approval voting: Who gets ruled in and ruled out," Electoral Studies, vol. 25, pp. 287-305, 2006.

[23] C.-J. Lin and W.-W. Wu, "A causal analytical method for group decision-making under fuzzy environment," Expert Systems with Applications, vol. 34, pp. 205-213, 2008.

[24] K. Gerling, H. P. Grüner, A. Kiel, and E. Schulte, "Information acquisition and decision making in committees: A survey," European Journal of Political Economy, vol. 21, pp. 563-597, 2005.

[25] P. J. Coughlin, Probabilistic voting theory: Cambridge University Press, 1992.

(C) Liu et al.; Licensee Bentham Open.

This is an open access article licensed under the terms of the Creative Commons Attribution Non-Commercial License (http://creativecommons.org/licenses/by-nc/3.0/) which permits unrestricted, non-commercial use, distribution and reproduction in any medium, provided the work is properly cited. 